\title{
Apoptotic Effects of Mount Bulgar Viper (Montivipera bulgardaghica) PLA2 and SVMPs Venom Peptide fractions on HeLa and A549 Cancer Cells ${ }^{\dagger}$
}

\author{
Yalcin Erzurumlu 1, Daniel Petras 2,3, Bayram Goçmen 4, Benjamin-Florian Hempel 2, Paul Heiss ${ }^{2}$, \\ Mehmet Zülfü Yildiz ${ }^{5}$, Roderich D. Süssmuth ${ }^{2}$ and Ayse Nalbantsoy ${ }^{6, *}$ \\ 1 Department of Biochemistry, Faculty of Pharmacy, Ege University, Izmir 35100, Turkey \\ 2 Institut für Chemie, Technische Universitat Berlin, Strasse des 17. Juni 124, Berlin 10623, Germany \\ 3 Skaggs School of Pharmacy \& Pharmaceutical Sciences, University of California-San Diego, PSB 4231, \\ Gilman Drive, La Jolla, CA 9500, USA \\ 4 Zoology Section, Department of Biology, Faculty of Science, Ege University, Bornova, Izmir 35100, Turkey \\ 5 Zoology Section, Department of Biology, Faculty of Arts and Science, Adiyaman University, \\ Adiyaman 02040, Turkey \\ 6 Department of Bioengineering, Faculty of Engineering, Ege University, Izmir 35100, Turkey \\ * Correspondence: analbantsoy@gmail.com; Tel.: +90-536-565-1895 \\ + Presented at the 2nd International Conference on Natural Products for Cancer Prevention and Therapy, \\ Kayseri, Turkey, 8-11 November 2017.
}

Published: 14 November 2017

\begin{abstract}
Snake venoms are extremely important for alternative medicine applications and discovery of new bioactive molecules. Especially in the recent years, there are promising studies in the literature on the development of new therapies for targeting the cancer with the use of snake venom peptides. In the present study, PLA2 and SVMPs venom peptide fractions of M. bulgardaghica were investigated for their apoptotic effects on human cervix adenocarcinoma cell line, HeLa and human lung adenocarcinoma cell line, A549. The cytotoxicity of crude venom was assessed by MTT assay with IC50 values of $4.34 \pm 0.19$ and $2.60 \pm 0.18 \mu \mathrm{g} / \mathrm{mL}$ against HeLa and A549 cells, respectively. Following treatment, the cells with fractions for $48 \mathrm{~h}$, the percentage of apoptotic cells was quantified by Annexin V/PI double staining assay and analyzed with flow cytometry. Our results show that, M. bulgardaghica venom fractions weren't significantly triggered apoptotic effect on cells but affected the cell cycle in PI staining experiments. As a result, snake venom peptides might have potential use in the treatment of cancer with new drug targeting techniques.
\end{abstract}

Keywords: M. bulgardaghica; venom; apoptosis; HeLa; A549

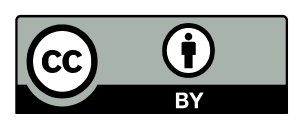

(C) 2017 by the authors. Licensee MDPI, Basel, Switzerland. This article is an open access article distributed under the terms and conditions of the Creative Commons Attribution (CC BY) license (http://creativecommons.org/licenses/by/4.0/). 